Originalveröffentlichung in: Kay Junge, Daniel Šuber, Gerold Gerber (Hg.), Erleben Erleiden Erfahren. Die Konstitution sozialen Sinns jenseits instrumenteller Vernunft, Bielefeld 2008, S. 357-371

\title{
Sakralkönigtum und Gemeinschaftskunst. Der Alte Orient und das Politische
}

JAN ASSMANN

Was das Politische angeht, hat der Alte Orient einen schlechten Ruf im europäischen Gedächtnis. ${ }^{1}$ Bis zur Entzifferung der originalen Quellen im neunzehnten Jahrhundert war Europa auf die biblischen, griechischen und lateinischen Nachrichten und damit auf die Außenansicht der altorientalischen Reiche angewiesen, und diese Außenansicht ist nicht sehr einladend. Mesopotamien, also >Babel< und >Ninive $<$, erscheinen in der Bibel als Inbegriff von Hybris, Willkürherrschaft und moralischer Verderbtheit; Ägypten ist das Haus der Knechtschaft und wird als Inbegriff von Unterdrückung, Schinderei, Zauberei und Götzendienst geschildert. Den Griechen tritt der Orient vor allem in der persischen Variante als >orientalische Despotie` entgegen, als gesetzlose Willkürherrschaft eines Einzelnen oder einer Clique über ein unterdrücktes Volk. Freiheit, Autonomie, politische Selbstbestimmung erscheinen sowohl in der Bibel als auch bei den Griechen als Kriterium der Selbstabgrenzung von den Reichen des Orients. Ägypten kommt bei einigen eher konservativen Staatsdenkern wie Isokrates, Platon und Hekataios von Abdera allerdings sehr viel besser weg. In der Gegenüberstellung von orientalischer Despotie, die auf Willkür gründet, und westlicher Politik, die auf Gesetz und Verfassung gründet, steht Ägypten für einen dritten Weg, der Monarchie und Gesetz verbindet (vgl. 2000: Kap. III.3). Im Ganzen aber ist das Image des Alten Orients, was das Politische angeht, eher schlecht. Stand er früher für das Gegenbild guter politischer Ordnung, so wird ihm heute $>$ das Politische (überhaupt abge-

1 Dieser Beitrag geht auf die von Bernd Giesen organisierte \Konstanzer Meisterklasse 2003 mit Mary Douglas und Shmuel N. Eisenstadt zurück, die unter dem Thema >Religion and Politics« stand (vgl. Giesen/Šuber 2005). 
sprochen, das im eigentlichen, noch heute gültigen Sinne, wie Christian Meier (1989) gezeigt hat, erst mit den Griechen entstand. ${ }^{2}$

Das Politische, das ist nach unserem Verständnis ein Raum öffentlichen Handelns und Entscheidens im Dienste der Polis und des Gemeinwohls, im Gegensatz zu den privaten Räumen des Handelns in eigener Sache. Ein Raum öffentlichen Handelns und Entscheidens setzt die Mitbestimmung freier Bürger voraus. Wenn ein Einzelner diesen Raum stellvertretend für alle besetzt und den Untertanen jede Möglichkeit der Mitbestimmung genommen ist, dann kann von dem Politischen nicht mehr die Rede sein. Zugleich mit dem Raum des Politischen und den Möglichkeiten bürgerlicher Mitbestimmung entsteht das Politische als Diskurs, zentriert um die Fragen nach der besten Verfassung, den besten Gesetzen und den Grundlagen von Herrschaft und Gerechtigkeit. Wenn jeder mitreden kann, müssen objektive Maßstäbe für die Richtigkeit und Gültigkeit von Entscheidungen gefunden werden. Das heißt Gerechtigkeit. Diesen Diskurs kann es nicht geben, wo alle diese Fragen schon vorab im Sinne der monarchischen Totalrepräsentation geklärt sind.

Der Alte Orient hat, soweit ich sehe, keine Alternativen zu der offenbar als einzig denkbar geltenden monarchischen Verfassung hervorgebracht. Vermutlich hat es auch keine Diskurse über die beste Verfassung gegeben. Bürgerliche Mitbestimmung wäre für Ägypten und Mesopotamien keine denkbare Alternative gewesen, weil die dafür notwendigen großräumigen Institutionen, Infrastrukturen und Kommunikationskanäle nicht existierten. Auch Rom hörte in dem Moment auf, eine Republik zu sein, als die dafür optimale Betriebsgröße allzu drastisch überschritten war, und die europäischen Staaten kehrten erst dann zu dem Politischen im griechischen Sinne zurück, als die für bürgerliche Mitbestimmung erforderlichen Infrastrukturen möglich geworden waren. Erst heute leben wir in einer Welt, in der sich die Demokratie als beste Verfassungsform und der Nationalstaat als optimale Betriebsgröße durchzusetzen begonnen haben. Freilich, nachdem das Politische als Diskurs einmal in die Welt gekommen war, war es, solange die klassischen Sprachen lesbar und die Texte zugänglich blieben, daraus nicht mehr wegzudenken und so kann es sich manchen so darstellen, als sei erst mit den Griechen das Politische in die Welt gekommen und seitdem nicht mehr daraus verschwunden.

Nachdem nun aber durch die Entzifferung der alten Schriften die originalen Quellen zugänglich geworden sind, sind wir nicht mehr ausschließlich auf die biblische und klassische Außenansicht des Alten Orients und seiner politischen Kultur bzw. Barbarei angewiesen, sondern können uns auch über die Innenansicht dieser Welt Aufschlüsse verschaffen. Das möchte ich hier in aller gebotenen Kürze versuchen und mich dabei weitgehend auf das Alte Ägypten beschränken. In seiner Innenansicht

2 Einen weiteren Begriff des Politischen vertritt Raaflaub (1993). 
stellt sich der ägyptische Staat (und ich glaube, man kann das verallgemeinern und sagen: der altorientalische Staat) als ein Institut der Gerechtigkeit dar. `Ma'at`, also Recht, Wahrheit, Ordnung in Ägypten, `kittu< und >mescharu, also Recht und Gerechtigkeit in Mesopotamien, und saschar, Gerechtigkeit und Wahrheit in Altiran sind die zentralen Begriffe aller politischen Diskurse, die es auch hier gegeben hat, auch wenn sie nicht um die Frage nach der besten Verfassung, sondern eher um die Grundlagen von Herrschaft und Gemeinschaft kreisten.

Die zentrale Aufgabe des ägyptischen Staates (bzw. quellensprachlich: des ägyptischen Königs, also Pharaos) war es, auf Erden Gerechtigkeit einzusetzen und das Unrecht zu vertreiben (vgl. Assmann 1990). Damit war die Errichtung einer Rechtssphäre gemeint, in der rohe Gewalt ausgeschlossen war und in der vor dem Gesetz alle gleich, das heißt die Unterschiede zwischen stark und schwach, arm und reich aufgehoben waren. Das Ideal der Rechtsprechung wurde weniger darin gesehen, die Übeltäter zu bestrafen als dem Schwachen gegenüber dem Starken zu seinem Recht zu verhelfen, für die Witwen und Waisen als die sprichwörtlich Entrechteten einzustehen, zwischen streitenden Parteien zu schlichten und die soziale Harmonie herzustellen und aufrecht zu erhalten. Der ägyptische Staatsmythos, der Mythos von Horus und Seth, erzählt, wie der schwache Knabe Horus, der legitime Sohn des ermordeten Osiris, und der starke Gewalttäter Seth, der Mörder seines Bruders Osiris, um die Nachfolge im Königsamt streiten. Nach langem Rechtstreit erhält Horus den Thron, Recht triumphiert über Gewalt, aber Seth wird anderweitig abgefunden und die Streitenden versöhnen sich, weil Recht ohne Gewalt hilflos ist, so wie Gewalt ohne Recht barbarisch ist. ${ }^{3}$ Das Recht bedarf, wie es schon Blaise Pascal in einer berühmten Passage seiner >Pensées〈 feststellte, der Gewalt zu seiner Durchsetzung.

"Die Gerechtigkeit ohne Gewalt ist kraftlos, ohnmächtig; Gewalt ohne Gerechtigkeit ist tyrannisch. Gerechtigkeit ohne Gewalt wird nicht anerkannt, weil es immer Bösewichte gibt; Gewalt ohne Gerechtigkeit wird angeklagt. Man muß also Gerechtigkeit und Gewalt zusammenstellen, damit, was gerecht und angemessen auch stark und kräftig, was stark und kräftig auch gerecht und angemessen ist.

Da man nicht hat erreichen können, daß das Gerechte und Angemessene auch stark und kräftig ist, hat man dafür gesorgt, daß das Starke und Kräftige gerecht und angemessen ist« (Derrida 1991: 23).

Als den zentralen politischen Diskurs im Alten Ägypten möchte ich die sog. ২Klagen ansprechen (Junge 1977). Sie bilden den Ort, an dem in der altägyptischen Welt über die Fragen der Herrschaft, Gemeinschaft und Gerechtigkeit nachgedacht wird. Das sind Texte, in denen ein Einzelner ent-

3 Vgl. zu diesem Mythos Frankfort (1948) und Griffiths (1960). 
weder in Form eines Selbstgesprächs oder gegenüber einer höheren Instanz den Zustand einer Welt beklagt, aus der Gerechtigkeit, Herrschaft und Gemeinschaft verschwunden sind. Diese drei gehören offenbar untrennbar zusammen. Keine Herrschaft ist ohne Gerechtigkeit und Gemeinschaft, keine Gerechtigkeit ohne Herrschaft und Gemeinschaft, keine Gemeinschaft ohne Herrschaft und Gerechtigkeit denkbar. Statt >Herrschaft müssen wir überall >Königtum einsetzen, das wird, wie schon gesagt, alternativlos gedacht. Der Grundsatz aber, dass keine Herrschaft ohne Gerechtigkeit und Gemeinschaft denkbar ist, entzieht schon einmal dem Klischee der orientalischen Despotie der Boden. Dass keine Gerechtigkeit und Gemeinschaft ohne Herrschaft bzw. Königtum denkbar sei, das allerdings steht nun unserem Begriff des Politischen diametral entgegen. Man könnte sagen, dass die große Leistung der Griechen darin besteht, diesen Nexus aufgebrochen zu haben.

In der Tat stellt der Nexus von Gerechtigkeit und Königtum das Grundprinzip der altorientalischen Staaten dar, das daher am Angemessensten als >Rechtskönigtum $<$ zu bezeichnen ist. Der König legitimiert sich dadurch, dass er die genannte Rechtssphäre herstellt und sich an sie bindet. Seine vornehmste Aufgabe besteht darin, die Gesetze zu erlassen und in Kraft zu setzen. Die Gesetze bilden das geltende Recht, und die Gerechtigkeit stellt eine übergeordnete Norm dar, an der sich die Gesetze zu orientieren und zu messen haben. Gerechtigkeit umfasst mehr als das Recht, sie umfasst auch Erbarmen, Amnestie, Rechtsverzicht in Fällen, wo die blinde Durchsetzung des Rechts die soziale Harmonie stören würde. Gerechtigkeit ist eine rettende Instanz, sie hat in erster Linie, wir haben es schon gesehen, die Aufgabe, die Schwachen vor der Unterdrückung durch die Starken und die Armen vor der Ausbeutung durch die Reichen zu schützen.

Im Grunde läuft die Idee einer »rettenden Gerechtigkeit« (Assmann/ Janowski/Welker 1998), die ihre Aufgabe darin sieht, die Schwachen vor den Starken zu schützen, auf eine Gleichsetzung von >starkı und `böse ২ hinaus. Nietzsche hat das bekanntlich als eine speziell jüdisch-christliche Errungenschaft angesehen und als »Ressentiment « und »Sklavenmoral« gedeutet (Nietzsche 1999). Max Weber ist ihm darin mit großer Zustimmung gefolgt (Fleischmann 1981). Damals waren die ägyptischen und vorderorientalischen Quellen noch unzureichend erschlossen. Inzwischen ist deutlich geworden, dass es sich hier um ein gesamtorientalisches Gerechtigkeitsdenken handelt, an dem die jüdisch-christliche Bibel lediglich auf ihre besondere Weise Anteil hat. Von Sklavenmoral kann überhaupt keine Rede sein. Auch diese Einschätzung zeigt nur wieder, wie fremd uns das Prinzip der rettenden Gerechtigkeit geworden ist.

Diesen Vorstellungen von Staat und Gerechtigkeit liegt eine negative Anthropologie zugrunde. Gäbe es den Staat nicht, der auf Erden für Gerechtigkeit sorgt, dann würde das Unrecht herrschen, dann würden die 
Starken die Schwachen unterdrücken und die Reichen die Armen ausbeuten. Das wird als Naturzustand vorausgesetzt. Wenn es als die erste Aufgabe des Königs angesehen wird, die >Ma'at< auf Erden zu verwirklichen und das Unrecht daraus zu vertreiben, dann wird unterstellt, dass ohne den König auf Erden das Unrecht herrscht. Diese Anthropologie finden wir auch anderswo, z.B. im >Arthashastra<, der Staatslehre des klassischen Indien, deren Lehren Max Weber als »nacktesten Machiavellismus« bezeichnete (Weber 1978: 65f., 145f.). ${ }^{4}$ Nur durch den Staat gelingt dem Menschen die Heraushebung aus dem Chaos der subhumanen Sphäre ewiger gegenseitiger Zerfleischung, in das er im Zustand der Herrschaftslosigkeit (>arajaka<) wieder zurückfällt: »Gäbe es auf Erden keinen König, der den züchtigenden Stock trägt, dann würden die Starken die Schwachen aufspießen und braten wie Fische«. ${ }^{5}$

Dem Staat, d.h. dem Krieger- und Fürstenstand ( $>$ kshatriya $)$, fällt in diesem Weltbild eine sehr umfassende Schutzpflicht zu, nämlich die, eine Ordnung des Zusammenlebens aufzubauen und zu garantieren, welche Menschentum (>manusha ) überhaupt erst möglich macht. Aus der pessimistischen Anthropologie und der Fiktion eines chaotischen Naturzustands ergab sich eine umfassende Ermächtigung zum Gewaltgebrauch und zur Aggressivität. In der abendländisch-christlichen Tradition wird die pessimistische Anthropologie seit Augustinus mit der Doktrin der Erbsünde begründet. So taucht das indische Bild vom >Gesetz der Fische` (১matsyanyaya $)$ auch in der mittelalterlichen Staatslehre auf: »Überall unterdrükken die Starken die Schwachen und die Menschen sind wie die Fische im Meer, die sich gegenseitig verschlingen«. Dieser Satz steht im Gesetzbuch Friedrichs II. von Hohenstaufen aus dem Jahre 1231. Durch den Sündenfall fassten die Menschen

"gegeneinander Hass, trennten den nach natürlichem Recht gemeinsamen Besitz der Dinge und [...] scheuten sich nicht, auf Streitigkeiten einzugehen. So [...] wurden die Fürsten der Welt gewählt, damit durch sie die Freiheit zu Verbrechen eingeschränkt werden könne« (Borst 1979: 285ff.).

$\mathrm{Zu}$ den anthropologisch pessimistischen Staatstheoretikern gehört auch Thomas Hobbes mit seiner Vorstellung von der wölfischen Natur des Menschen (’homo homini lupus $\varsigma$ ) und seiner Theorie des Naturzustands als eines Kriegs aller gegen aller (〉bellum omnium contra omnes $<$ ). Carl Schmitt hat diesen Zusammenhang von Staatslehre und Anthropologie in der 2. Auflage seines Buches `Der Begriff des Politischen` (1932) sehr klar formuliert:

4 Vgl. zum >Arthashastra< auch Louis Dumont, der feststellt: »Die oft betonte Parallele zu Machiavelli ist durchaus nicht unsinnig « (1976: 353).

5 Originalquelle: Mahabharata XII, 67, 16 (Dumont 1976: 351). 
»Man könnte alle Staatstheorien und alle politischen Ideen auf ihre Anthropologie prüfen und danach einteilen, ob sie, bewußt oder unbewußt, einen svon Natur bösen` oder einen >von Natur guten« Menschen voraussetzen« (Schmitt 1979: 59ff.).

Wenn er dann feststellt, dass »alle echten politischen Theorien (den Menschen) als >böse<, d.h. als keineswegs unproblematisches, sondern als >gefährliches` und dynamisches Wesen voraussetzen« (ebd.), dann ist klar, dass für ihn auch die altorientalischen Staatslehren >echte< politische Theorien darstellen. Im gleichen Sinne wie Schmitt, darin waren die beiden sich einig, erkennt auch Leo Strauss in der Gefährlichkeit des Menschen die Quelle seiner »Herrschaftsbedürftigkeit« (Strauss 1998: 114f.). So uralt diese politischen Ideen sind, so schwer sind sie zu überwinden. Noch im Jahre 2005 schrieb Rudolf Burger:

»Der Liberalismus, geborgen und befangen in einer Welt der Kultur, vergißt das Fundament der Kultur, den Naturzustand, das heißt die menschliche Natur in ihrer Gefährlichkeit und Gefährdetheit. Mit anderen Worten: Er vergißt die Wolfsnatur des Menschen« (Burger 2005: 1041).

Nun muss man aber, was die Anthropologie angeht, einen wichtigen Unterschied zwischen der ägyptischen und der Hobbes'schen Staatslehre berücksichtigen. Hobbes spricht dem Menschen alle angeborene Sozialität ab. Für ihn ist der Mensch ein ausschließlich auf Selbsterhalt und Lustgewinn ausgerichtetes Individuum, das sich mit anderen Menschen nur in Verfolgung seiner eigenen Interessen verbindet. Für die Ägypter dagegen ist der Mensch (wie für Aristoteles) ein von Haus aus auf Bindung angelegtes Wesen, ein sanimal sociale`, das nur in Gemeinschaft mit anderen leben kann. Diesem natürlichen Bindungsbedürfnis steht zwar ein Trieb nach Macht und Selbstdurchsetzung entgegen, aber dieser Trieb ist durch Erziehung formbar. Die Ägypter gehen also davon aus, dass der Mensch nicht nur durch den äußeren Zwang der politischen Herrschaft, d.h. durch Androhung von Gegengewalt, sondern auch und vor allem durch Erziehung dazu gebracht werden kann, das Recht zu achten und den Schwachen zu schonen. Die Gerechtigkeit ist zwar nicht von Natur aus im Menschen angelegt, aber er ist von Natur aus auf Gemeinschaft und damit auf Gerechtigkeit und Herrschaft hin angelegt, so dass er $\mathrm{zu}$ ihr erzogen werden kann, von wenigen unverbesserlichen Egoisten abgesehen.

Dieser Erziehung und Unterweisung dienen die Texte, die ich für das Alte Ägypten als eine Form politischen Diskurses reklamieren möchte. Es handelt sich um die Lehren einer Gemeinschaftskunst, die den Menschen zum Mitmenschen und zum harmonischen und erfolgreichen Zusammen- 
leben mit Anderen erziehen möchte. ${ }^{6}$ Wir dürfen den Begriff des Politischen nicht mit Herrschaft gleichsetzen. Das Politische hat immer zwei Dimensionen, die vertikale der Herrschaft und die horizontale der Gemeinschaft. Die eine ist immer in der anderen mitgedacht. Der ägyptische Begriff von Gemeinschaft beruht auf dem Prinzip der ivertikalen Solidaritätr. $\mathrm{Um} \mathrm{das} \mathrm{zu} \mathrm{erklären,} \mathrm{muss} \mathrm{ich} \mathrm{noch} \mathrm{einmal} \mathrm{auf} \mathrm{den} \mathrm{ägyptischen}$ Staatsmythos, den Mythos von Horus und Seth, zurückkommen. Dieser Mythos beruht auf zwei Konstellationen: der Feindschaft zwischen den Brüdern Osiris und Seth, und dem Bündnis zwischen Vater und Sohn, Osiris und Horus. Dies Bündnis besteht über die Todesschwelle hinweg und verhilft dem toten Vater nicht nur zu seinem Recht, sondern auch zu einer Art von Auferstehung. Indem der Sohn auf Erden seinen Platz in der Gesellschaft übernimmt und für ihn eintritt, stellt er seine Ehre und damit einen wichtigen Aspekt seiner sozialen Persönlichkeit wieder her. Auf diesem Vater-Sohn-Bündnis beruhen in Ägypten Staat und Kultur. Es stellt die genaue Umkehrung der Freudschen Konstruktion der ödipalen Konstellation dar. Wie bei Freud Vatermord und Bruderbund, so gehören bei den Ägyptern Brudermord und Vaterbund zusammen. So wie der Bruderbund als Prinzip einer Gemeinschaftskunst auf dem Gedanken einer `horizontalen` Solidarität zwischen Gleichen, so fordert der Vaterbund die >vertikaleく Solidarität zwischen Ungleichen: Höheren und Niederen in der Sozialdimension, Toten und Lebenden in der Zeitdimension. Vertikale Solidarität bedeutet Verantwortung und Schutz von oben nach unten, Loyalität und Gehorsam von unten nach oben. Während horizontale Solidarität die Ungleichheit zwischen den Menschen aufheben will, setzt vertikale Solidarität sie als naturgegeben und unaufhebbar voraus und will nur ihre Folgen mildern: durch soziale Normen, die Wohltätigkeit und Armenpflege, Erbarmen und Verantwortung einschärfen im Sinne von Verpflichtungen, die mit der Zugehörigkeit zur Hierarchie verbunden sind. Die Starken werden nicht geschwächt und die Schwachen nicht gestärkt, aber den Ersteren wird Verantwortung für die letzteren, den Letzteren Loyalität gegenüber den Ersteren eingeschärft.

Diese Konzeption einer vertikalen Solidarität ist uns gründlich fremd geworden. So kann es uns als selbstverständlich erscheinen, das Politische mit dem horizontalen Prinzip der brüderlichen Gleichheit zu identifizieren. Ich will hier auch in keiner Weise für das vertikale und gegen das horizontale Prinzip der Vergemeinschaftung plädieren. Ich wende mich nur gegen die Vereinseitigung, durch die der Alte Orient mit seinen politischen Errungenschaften von vornherein aus dem Blick gerät. Immerhin sind hier die ersten politischen Ordnungen großen Stils entstanden, wobei Ägypten

6 Zum Begriff der "Gemeinschaftskunst« vgl. Assmann/Krippendorff/ Schmidt-Glintzer (2006).

7 Diesen Begriff habe ich in meinem Buch `Ma'at< (Assmann 1990) näher erläutert. 
als der erste große Flächenstaat der Geschichte gelten kann. Die Griechen hatten zwar, wie Christian Meier betont, keine Griechen vor sich, aber sie hatten die altorientalischen Großreiche und Hochkulturen vor Augen, von deren Beispiel sie sich, wenn sie ihm schon nicht folgen wollten, doch immerhin abkehren konnten. Die Alten Ägypter hatten nur die Prähistorie vor sich.

Wenn uns schon das Prinzip der vertikalen Solidarität fremd geworden ist, dann gilt das in einem noch ganz anderen Maße für das, was ich die politische Theologie der altorientalischen Reiche nennen möchte, also die Formen, in denen die beiden Achsen der Politik, die Herrschaft und die Gemeinschaft, religiös interpretiert bzw. sakralisiert wurden. Im Alten Orient kann man zwischen Religion und Politik bzw. zwischen Kirche und Staat nicht unterscheiden. Weder das Politische noch das Religiöse sind als eigengesetzliche Sphären menschlichen Denkens und Handelns ausdifferenziert. Das ist einer der entscheidenden und durchaus einleuchtenden Gründe Christian Meiers, erst den Griechen die Erfindung des Politischen zuzuschreiben. Allerdings ist diese Ausdifferenzierung mindestens ebenso wie eine griechische auch eine biblische Errungenschaft; vor allem aber ist sie in der abendländischen Geschichte immer wieder rückgängig gemacht worden, so dass die altorientalische politische Theologie allen biblischen und griechischen Errungenschaften zum Trotz durch das ganze Mittelalter und bis weit in die Neuzeit hinein kaum etwas von ihrer Aktualität verloren hat. Gerade in den Ideen der politischen Theologie ist der Alte Orient, wie zu zeigen sein wird, im politischen Denken des Abendlandes am intensivsten lebendig geblieben.

Beginnen wir mit der Achse der Herrschaft. Wieder konzentriere ich mich auf Ägypten und stelle anheim, wie weit sich diese Dinge auf Mesopotamien übertragen lassen (vgl. Sigrist 2004). Als erstes ist hier festzustellen, dass die Herrschermacht als solche als etwas Heiliges und Übermenschliches gilt. Sie kann nicht von Menschen ausgeübt werden. In Mesopotamien gilt >lugalı, der König, als eine besondere, gewissermaßen überlebensgroße und götternahe Art Mensch, in Ägypten ist >Pharao schlichtweg ein Gott (vgl. Frankfort 1948, Gundlach/Weber 1992, O'Connor/Silverman 1995, Gundlach/Klug 2004). Nichts könnte unserem ebenso wie dem griechischen, aber auch dem biblischen politischen Denken fremder sein. Herrschen gilt den Ägyptern als Fortsetzung der Schöpfung unter den Bedingungen der geschaffenen Welt. Nachdem der Sonnengott die Welt teils geschaffen, teils sich in sie verwandelt hat, und nachdem er diese aus ihm hervorgegangene Welt zusammen mit anderen Göttern beherrscht hat, zog er sich samt seinem Pantheon in den Himmel zurück und delegierte die irdische Herrschaft an den Pharao. Das wird in einem sehr zentralen und kanonischen Text folgendermaßen ausgedrückt: 
»Re (der Schöpfer- und Sonnengott) hat den König eingesetzt auf der Erde der Lebenden für immer und ewig, um den Menschen Recht zu sprechen und die Götter zufriedenzustellen, um die Ma'at zu verwirklichen und das Unrecht zu vertreiben. Er gibt den Göttern Gottesopfer und den Toten Totenopfer« (Assmann 1970; 1983: 48f.; 1990: 205ff.; Betrò 1990).

Hier sind die Aufgaben des Staates noch einmal zusammengefasst. Er muss die >Ma'at` verwirklichen und deren Gegenteil, die >Isfetく (Lüge, Unrecht, Chaos), vertreiben, dadurch, dass er den Menschen Recht spricht und die Götter mit Opfern zufrieden stellt. Recht und Kult also sind die vornehmsten Aufgaben des Königs. Dafür ist er auf Erden eingesetzt.

Pharao bildet also nicht allein die Spitze der politischen Pyramide. Er ist eine Verweisfigur, die auf einen noch Höheren hindeutet: den Sonnenund Schöpfergott, der ihn als seinen Sohn auf Erden eingesetzt hat, damit er das Werk der Weltinganghaltung dort im Rahmen der Menschenwelt durchführe wie der Sonnengott im Rahmen der Götterwelt. Hier treffen wir nun, als Herzstück der ägyptischen politischen Theologie, wieder auf das Vater-Sohn-Bündnis, auf dem, wie ich sagte, in Ägypten Staat und Kultur beruhen. Pharao ist der Sohn in dieser Konstellation, der allen Göttern als seinen Vätern und Müttern gegenübertritt. Er ist ein Gott, aber ein Sohn-Gott, der sein Herrscheramt nur in Verbindung mit seinem Vater und der Götterwelt ausüben kann. Als Sohn-Gott trägt er den Namen des paradigmatischen Sohnes in der ägyptischen Götterwelt, Horus, des Sohnes und Rächers des erschlagenen Osiris. Der Gott Horus verkörpert sich in jedem regierenden König; das ist die ägyptische Variante jener politischen Theologie, die Ernst Kantorowicz (1990) als die Lehre von den zwei Körpern des Königs bekannt gemacht hat. Auf Darstellungen sieht man oft den König begleitet von einem Doppelgänger, seinem >Kaく, der den Horus-Namen auf dem Kopf trägt. Der >Königs-Ka des Königs, der neben seinem vergänglichen Individualkörper einhergeht. Während im Alten Reich, dem ersten ägyptischen Staat des dritten Jahrtausends vor Christus, die Göttlichkeit des Pharao herausgestellt wurde, der als Gott auf Erden herrschte, kam es im Mittleren und Neuen Reich mehr auf das Repräsentationsverhältnis Pharaos zum Schöpfer- und Sonnengott an, und die häufigste Umschreibung seiner Rolle war nun die eines Bildes oder lebenden Bildes Gottes (z.B. >Tutanchamun $=$ >lebendes Ebenbild Amuns $\varsigma$ ) (Hornung 1967: 123ff., Ockinga 1984).

Die Konzeption Pharaos als Sohnes und Bildes Gottes ist in der Bibel bekanntlich auf die radikalste Weise demokratisiert worden. Die Bibel erklärt den Menschen schlechthin zum Bilde Gottes, das Prädikat der Sohnschaft aber wird dem auserwählten Volk zugesprochen. In dieser Rolle tritt Israel an die Stelle Pharaos. Aber auch das mesopotamische Königsmodell wird in der Bibel in gleicher Weise demokratisiert und auf die Beziehung Gottes zu seinem Volk umgedeutet. In Mesopotamien beruht die Vorstel- 
lung der Beziehung zwischen König und Götterwelt auf dem Modell der heiligen Hochzeit. Einmal im Jahr steigt der König in das Brautgemach auf der Spitze des Tempelturms und vermählt sich dort mit der Göttin Inanna bzw. Ischtar (Steinkeller 1999: 103ff.). Daraus wird in der Bibel die Metapher der Brautschaft Israels zu seinem Gott.

Andere Aspekte des altorientalischen Königsbildes aber werden nun dem biblischen Gott zugeschrieben. Dazu gehört in allererster Linie der zentrale Aspekt des Gesetzgebers. Aus der Gerechtigkeit, die der König auf Erden verbreiten soll, wird jetzt Gottes Gerechtigkeit. Damit wird nun auch erstmals zwischen den Sphären des Politischen und des Religiösen strikt unterschieden. Das gilt zwar nicht für die Königszeit und die Königspsalmen, in denen der König noch genau wie in Ägypten als Sohn und Repräsentant Gottes angesprochen wird, aber für das Deuteronomium, das dann zum Ausgangspunkt einer ganz neuen und bis heute bestimmenden Konzeption von Religion und Politik wird. Jetzt wird das Königtum säkularisiert und das Religiöse allein auf Gott bezogen. Die religiöse Dimension des Königtums wird in der Figur des Messias eschatologisiert, d.h. aus der Geschichte ausgelagert und auf das Ende der Geschichte verschoben. Bis dahin ist Gott in der Menschenwelt durch keinen Herrscher repräsentierbar. Die politische Zentralidee des Alten Orients, die Beziehung zwischen Gott und Herrscher im Sinne der Sohnschaft oder Repräsentation, also die Vorstellung der Immanenz des Göttlichen im Herrscher, erscheint nun als Inbegriff des Heidentums. In dieser Form aber wird sich das Heidentum als praktisch unüberwindlich erweisen. Offenbar ist die Idee der göttlichen Immanenz im Herrscher von so verführerischer Strahlkraft, dass sie durch keine Aufklärung endgültig aus der Welt zu schaffen ist. ${ }^{8}$ In der politischen Theologie hat die altorientalische Konzeption des Politischen am längsten und kraftvollsten überdauert und immer wieder neue Auferstehungen gefeiert, sei es in der Form der wundertätigen Könige, des Kirchenstaats, des byzantinischen und französischen Cäsaropapismus, des Gottesgnadentums, des Herrschers als des Gesalbten Gottes oder in der Form des Kalifats und der politische Macht ausübenden Mullahs und Ayatollahs. ${ }^{9}$ Offenbar fällt es den Menschen schwer, das Politische und das Religiöse auseinander zu halten. Entweder wird das Religiöse abgeschafft und das Politische an seine Stelle gesetzt wie in den Totalitarismen des zwanzigsten Jahrhunderts oder das Politische wird dem Religiösen untergeordnet und gleichgeschaltet wie im islamistischen Gottesstaat, oder man

8 So verwendet, wie Martin Mulsow (2002) gezeigt hat, Daniel Georg Morhof (1639-1691) den Begriff stheologia politica in seiner >Theologia politica gentium (1662). Auch Erik Peterson (1935) scheint unter >politischer Theologie < diese heidnische Form verstanden zu haben, wenn er meinte, dass das Christentum mit seiner Trinitätslehre jede politische Theologie erledigt habe. Gemeint ist, dass sich die Trinität in Herrschaftsformen nicht abbilden lässt.

9 Siehe hierzu bahnbrechend Al-Azmeh/Bak (2004). 
hält wie etwa in Japan mitten im modernen Staat an der Immanenz des Göttlichen im Herrscher fest, denn dort sitzt wie im Alten Ägypten ein veritabler Gottessohn und Abkömmling der Sonnengottheit auf dem Thron.

Wie steht es aber mit der anderen Dimension des Politischen, der Gemeinschaft? Hier kommt nun der Begriff der `Gerechtigkeit, der andere Zentralbegriff des altorientalischen politischen Denkens, zum Tragen. Die Gerechtigkeit, ägyptisch >Ma'atı, ist es, die die Menschen zur Gemeinschaft verbindet. Wird auch diese Idee im Alten Orient sakralisiert, so wie die Idee des Herrschens? Die Antwort ist `ja und nein $<$. Ja, denn >Ma'at ist nicht nur ein abstrakter Begriff, sondern wird auch als eine Göttin personifiziert. Zweifellos kann man diese Idee nicht vom Reich des Religiösen ablösen. Außerdem haben wir gesehen, dass zur Sphäre der Gerechtigkeit, die der König auf Erden durchsetzen soll, auch der Kult gehört. Ähnlich steht es in Mesopotamien. Gerade in seiner Rolle als Gesetzgeber und Rechtspfleger ist der König den Göttern ganz besonders verantwortlich. Wenn man aber von hier auf die Bibel schaut, dann wird sofort deutlich, dass sich erst hier die wahre Sakralisierung der Gerechtigkeit und Gemeinschaft durchsetzt, wo Gott selbst als Gesetzgeber auftritt und die Gerechtigkeit von den Menschen einklagt. Hier treten Recht und Gerechtigkeit in die Mitte der Beziehung zwischen Gott und Menschen; der Kult tritt demgegenüber zurück. Das Rechttun, die Befolgung der Gesetze, wird im Judentum zum vornehmsten Gottesdienst. Mit dieser Sakralisierung der Gerechtigkeit geht die Sakralisierung der Gemeinschaft in der Idee des Gottesvolks einher. »Ihr sollt mir sein ein Königreich von Priestern und ein heiliges Volk«, sagt Gott in Ex 19.6.

Diese Vorstellung ist dem politischen Denken des Alten Orients fremd. Der biblischen Idee des Gottesvolks, überhaupt des >Volkes` ( $>$ am 'goj<), lässt sich hier kein politischer Begriff vergleichbarer Bedeutung zur Seite stellen. Der biblische Volksbegriff ist ein Einheits- und ein Differenzbegriff. Einmal geht es um die Einheit des Volkes jenseits der zwölf Stämme, zum anderen um die Erwählung Israels unter den Völkern. Die Bedeutung als Differenzbegriff wird immer wichtiger, je mehr die Stammesstruktur verblasst. Im gleichen Zusammenhang werden Gesetz und Gerechtigkeit zum entscheidenden Kriterium sowohl der Zugehörigkeit zum Gottesvolk als auch der Differenz zu den anderen Völkern. Der Vorgang spielt sich ab in einer Zeit, in der die Völker der Mittelmeerwelt anfangen, um den Rang des Staatswesens mit den besten Gesetzen und der besten Verfassung zu wetteifern. Der unverhohlene Stolz des Deuteronomiums auf die unübertroffenen israelitischen Gesetze ist also kein Einzelfall; Ähnliches gilt für Sparta, Karthago, Rom. Zu diesem Rangstreit und den entsprechenden politischen Differenzbegriffen konnte es erst im ersten Jahrtausend vor Christus kommen, als die Staaten kleiner und vielfältiger wurden, die Kontakte und Konflikte sich intensivierten und sich jenseits 
dieser Vielfalt eine erste Form von kultureller, ökonomischer und später auch politischer Globalisierung anzubahnen begann, in der die Kulturen um ihr Überleben kämpfen mussten. Damit waren die Bedingungen für ganz neue Formen politischen Denkens geschaffen.

Nun hat aber, wie bereits betont, die weitere Geschichte weder an den griechischen, noch den biblischen Erfindungen bzw. Ausdifferenzierungen des Politischen festgehalten. Schon in der griechischen Welt wurde die altorientalische Idee des Rechtskönigtums und der herrscherlichen Rechtssouveränität wieder aufgegriffen und in Gestalt des Königs als >nomos empsychos` oder >lex animata<, also des verkörperten Gesetzes, auf den Begriff gebracht. ${ }^{10}$ Genau in dieser Rolle, wenn auch nicht in dieser Begrifflichkeit, waren schon die altorientalischen Könige aufgetreten. Mit dem christlichen Auszug aus dem jüdischen Gesetz und der historischen Besetzung der Messiasrolle durch Jesus von Nazareth war dann auch der repräsentativen politischen Theologie wieder Tür und Tor geöffnet. In seiner 1935 erschienenen Schrift \Der Monotheismus als politisches Problem - ein Werk, das kein Geringerer als Arnaldo Momigliano (1987: 153) als »the most remarkable book ever produced « über dieses Thema bezeichnete - vertrat Erik Peterson die These, das christliche Trinitätsdogma habe den

»Bruch mit jeder >politischen Theologie` vollzogen, die die christliche Verkündigung zur Rechtfertigung einer politischen Situation mißbraucht. Nur auf dem Boden des Judentums oder Heidentums kann es so etwas wie eine spolitische Theologie 'geben « (1935: 105). ${ }^{11}$

Das mag theologisch einleuchten; in der geschichtlichen Wirklichkeit war eher das Gegenteil der Fall. Die Herrscher der nachantiken, christianisierten Staatenwelt übertrafen sich als allerchristlichste, allerkatholischste und allerorthodoxeste Majestäten geradezu an Prädikaten ihrer Gottgesandtheit, Gotterwähltheit, Gottgesalbtheit und kehrten in dieser Hinsicht auf massivste Weise zur politischen Theologie des Alten Orients zurück. Wenn man an die nicht gerade seltenen Königsmorde im Alten Orient denkt, so gingen sie in der Sakralisierung der Herrschaft womöglich sogar noch weiter als dieser.

Etwas Entsprechendes muss nun abschließend auch für den vielleicht wichtigsten Aspekt des Politischen festgestellt werden: die Gewalt. Um mit einer ebenso plakativen wie trivialen Feststellung zu beginnen: der Alte Orient kannte keine Religionskriege. Natürlich spielte die Religion auch in der Kriegsführung eine große Rolle. Die Divisionen des ägyptischen Heeres trugen Götternamen, die assyrischen und ägyptischen Kriege wur-

10 Zur Lehre vom >Lebenden Nomos` siehe Ehrhardt (1959: 168ff.) sowie Goodenough (1979).

11 Vgl. hierzu auch Schindler (1978). 
den im Namen und im Auftrag der Staatsgötter geführt, die Kriegsbeute landete zu großen Teilen in den Schatzhäusern der Tempel, aus denen die Kriege auch finanziert wurden. Trotzdem handelte es sich nicht um Religionskriege. $\mathrm{Zu}$ Religionskriegen kann es nur kommen in einem Raum, der durch die Unterscheidung zwischen wahrer und falscher Religion, wahren und falschen Göttern, wahrem und falschen Glauben strukturiert ist. Vorher und anderswo ist die politische und gerade auch die militärische Gewalt religiös verbrämt, gerahmt oder interpretiert, sogar ritualisiert und sakralisiert, aber nicht religiös motiviert. Die Götter werden für politische Zwecke in den Dienst genommen, aber nicht umgekehrt. Es geht um politische Interessen, nicht um religiöse. Religiöse Interessen sind gebunden an die Idee der offenbarten Wahrheit, die es durchzusetzen, der es Raum zu schaffen gilt. Der erste Religionskrieg der Geschichte ist der Makkabäerkrieg, nicht der Aufstand gegen Seleukos IV. Epiphanes, sondern der damit verbundene innerjüdische Bürgerkrieg gegen die hellenisierten Schichten und Städte des Judentums. Es ist zugleich der letzte jüdische Religionskrieg. Erst Christentum und Islam haben diese Tradition voll zur Entfaltung gebracht und weitergeführt. Religionskriege sind Kriege, die im Dienste und für die Sache Gottes geführt werden.

Es war ein Befreiungsschlag der Bibel, die altorientalische Einheit des Politischen und des Sakralen, von Herrschaft und Heil, aufzusprengen, aber diese Trennung ist immer wieder auf zweifache Weise rückgängig gemacht worden: entweder dadurch, dass sich die Kaiser und Könige wieder zu Repräsentanten Gottes auf Erden aufgeschwungen haben, oder dadurch, dass sich religiöse Instanzen mit politischer Gewalt ausgestattet oder verbündet haben. In dieser Form ist der Alte Orient bis heute nie ganz überwunden worden.

\section{Literatur}

Al-Azmeh, Aziz/Bak, János M. (Hg.) (2004). Monotheistic Kingship. The Medieval Variants. Budapest: Central European University Press.

Assmann, Jan (1970). Der König als Sonnenpriester. Ein kosmographischer Begleittext zur kultischen Sonnenhymnik in thebanischen Tempeln und Gräbern. Abhandlungen des Deutschen Archäologischen Instituts Abteilung Kairo. Bd. 7. Berlin: Achet Verlag.

Assmann, Jan (1983). Sonnenhymnen in Thebanischen Gräbern. Theben I. Mainz. Assmann, Jan (1990). Ma'at. Gerechtigkeit und Unsterblichkeit im Alten Ägypten. München: Beck.

Assmann, Jan (2000). Weisheit und Mysterium. Das Bild der Griechen von Ägypten. München: Beck. 
Assmann, Jan/Janowski, Bernd/Welker, Michael (Hg.) (1998). Gerechtigkeit. Richten und Retten in der abendländischen Tradition und ihren altorientalischen Ursprüngen. München: Fink.

Assmann, Jan/Krippendorff, Ekkehard/Schmidt-Glintzer, Helwig (2006). Ma'at - Konfuzius - Goethe. Drei Lehren für das richtige Leben. Frankfurt/Main: Insel.

Betrò, Marilina C. (1990). I testi solari del portale di Pascerientaisu (BN 2). SAQQARA III. Pisa.

Borst, Arno (1979). Lebensformen im Mittelalter. Frankfurt: Ullstein.

Burger, Rudolf (2005). »Der sterbliche Gott. Eine Bildbetrachtung«. In: Merkur.

Deutsche Zeitschrift für europäisches Denken 59. Jg. Nr. 679. S. 1032-1041.

Derrida, Jacques (1991). Gesetzeskraft. Der >mystische Grund der Autoritätর. Frankfurt/Main: Suhrkamp.

Dumont, Louis (1976). Gesellschaft in Indien. Die Soziologie des Kastenwesens. Wien: Europa-Verlag.

Ehrhardt, Arnold A.T. (1959). Politische Metaphysik von Solon bis Augustin.

Bd. 1: Die Gottesstadt der Griechen und Römer. Tübingen: J.C.B. Mohr.

Fleischmann, Eugène (1981). »Max Weber, die Juden und das Ressentiment«.

In: Schluchter, Wolfgang (Hg.): Max Webers Studie über das antike Judentum. Frankfurt/Main: Suhrkamp. S. 263-286.

Frankfort, Henri A. (1948). Kingship and the Gods. A Study of Ancient Near Eastern Religion as the Integration of Society and Nature. Chicago: The University of Chicago Press.

Giesen, Bernhard/Šuber, Daniel (Hg.) (2005). Religion and Politics. Cultural Perspectives. Leiden: Brill.

Goodenough, Erwin R. (1979). »Die politische Philosophie des hellenistischen Königtums«. In: Kloft, Hans (Hg.): Ideologie und Herrschaft in der Antike. WdF 528. Darmstadt: Wissenschaftliche Buchgesellschaft. S. 27-89.

Griffiths, John Gwyn (1960). The Conflict of Horus and Seth from Egyptian and Classical Sources. Liverpool: University Press.

Gundlach, Rolf/Klug, Andrea (Hg.) (2004). Das ägyptische Königtum im Spannungsfeld zwischen Innen- und Aussenpolitik im 2. Jahrtausend v. Chr. Wiesbaden: Harrassowitz Verlag.

Gundlach, Rolf/Weber, Hermann (1992). Legitimation und Funktion des Herrschers. Vom ägyptischen Pharao zum neuzeitlichen Diktator. Schriften der Mainzer Philosophischen Fakultätsgesellschaft Nr. 13. Stuttgart: Franz Steiner Verlag.

Hornung, Erik (1967). »Der Mensch als >Bild Gottes« in Ägypten«. In: Loretz, Oswald (Hg.): Die Gottebenbildlichkeit des Menschen. München: Kösel.

Junge, Friedrich (1977). »Die Welt der Klagen«. In: Assmann, Jan/Feucht, Eri$\mathrm{ka} / \mathrm{Grieshammer}$, Reinhard (Hg.): Fragen an die altägyptische Literatur (Gedenkschrift E. Otto). Wiesbaden: Reichert. S. 275-284.

Kantorowicz, Ernst H. (1990). Die zwei Körper des Königs. Eine Studie zur politischen Theologie des Mittelalters. München: dtv. 
Meier, Christian (1989). Die Entstehung des Politischen bei den Griechen. Frankfurt/Main: Suhrkamp.

Momigliano, Arnaldo (1987). On Pagans, Jews, and Christians. Middletown, Conn.: Wesleyan University Press.

Mulsow, Martin (2002). Moderne aus dem Untergrund. Radikale Frühaufklärung in Deutschland 1680-1720. Hamburg: Meiner.

Nietzsche, Friedrich (1999). »Zur Genealogie der Moral. Eine Streitschrift.« In: Ders.: Kritische Studienausgabe. München: de Gruyter. S. 257-289.

Ockinga, Boyo (1984). Die Gottebenbildlichkeit im Alten Ägypten und im Alten Testament. Wiesbaden: Harrassowitz.

O’Connor, David/Silverman, David P. (Hg.) (1995). Ancient Egyptian Kingship. Leiden: Brill.

Peterson, Erik (1935). Monotheismus als politisches Problem. Ein Beitrag zur Geschichte der politischen Theologie im Imperium Romanum. Leipzig: Hegner.

Raaflaub, Kurt A. (Hg.) (1993). Anfänge politischen Denkens in der Antike. Schriften des Historischen Kollegs. Kolloquien 24. München: Oldenbourg.

Sandall, Roger (2005). »Das Fortschrittsparadox. Über die Wurzeln des romantischen Primitivismus«. In: Merkur. Deutsche Zeitschrift für europäisches Denken 59. Jg. Nr. 679. S. 1042-1049.

Schindler, Alfred (Hg.) (1978). Monotheismus als politisches Problem? Erik Peterson und die Kritik der politischen Theologie. Studien zur evangelischen Ethik 14. Gütersloh.

Schmitt, Carl (1979). Der Begriff des Politischen. Berlin: Duncker und Humblot. Sigrist, Christian (Hg.) (2004). Macht und Herrschaft. Veröffentlichungen des Arbeitskreises zur Erforschung der Religions- und Kulturgeschichte des Antiken Vorderen Orients und des Sonderforschungsbereiches 492. Bd. 5. Münster: Ugarit.

Steinkeller, Piotr (1999). »On Rulers, Priests, and Sacred Marriage: Tracing the Evolution of Early Sumerian Kingship«. In: Watanabe, Kazuko (Hg.). Priests and Officials in the Ancient Near East: Papers of the Second Colloquium on the Ancient Near East. The Middle Eastern Culture Center in Japan. Heidelberg: Winter, S.103-137.

Strauss, Leo (1998). "Anmerkungen zu Carl Schmitt: Der Begriff des Politischen«. In: Meier, Heinrich (Hg.). Carl Schmitt, Leo Strauss und >Der Begriff des Politischen . Zu einem Dialog unter Abwesenden. Stuttgart: Metzler. S. 97-125.

Weber, Max (1978). Wirtschaft und Gesellschaft. Grundriß der verstehenden Soziologie. Bd. 2. Tübingen: Mohr. 